\title{
A Non-MHC Locus Essential for Autoimmune Type I Diabetes in the Komeda Diabetes-Prone Rat
}

\author{
Norihide Yokoi, ${ }^{\star}$ Masao Kanazawa, ${ }^{\ddagger}$ Kazuhiro Kitada, ${ }^{\star}$ Akihiko Tanaka, ${ }^{\ddagger}$ Yasunori Kanazawa, ${ }^{\S}$ Shigehiko Suda, ${ }^{\ddagger}$ Hisao Ito, ${ }^{\ddagger}$ \\ Tadao Serikawa, ${ }^{*}$ and Kajuro Komedall \\ *Institute of Laboratory Animals, Faculty of Medicine, Kyoto University, Kyoto 606-01, Japan; ${ }^{\ddagger}$ Third Department of Internal Medicine, \\ Tokyo Medical College, Tokyo 160, Japan; ${ }^{\S}$ Omiya Medical Center, Jichi Medical School, Omiya 330, Japan; and $\|^{D i v i s i o n}$ of Laboratory \\ Animal Science, Animal Research Center, Tokyo Medical College, Tokyo 160, Japan
}

\begin{abstract}
The Long-Evans Tokushima Lean (LETL) rat, characterized by rapid onset of insulin-dependent (type I) diabetes mellitus (IDDM), no sex difference in the incidence of IDDM, autoimmune destruction of pancreatic $\beta$ cells, and no significant $T$ cell lymphopenia, is a desirable animal model for human IDDM. We have established a diabetes-prone substrain of the LETL rat, named Komeda Diabetes-Prone (KDP) rat, showing a $100 \%$ development of moderate to severe insulitis within $220 \mathrm{~d}$ of age. The cumulative frequency of IDDM was $70 \%$ at $120 \mathrm{~d}$ of age, and reached $82 \%$ within $220 \mathrm{~d}$ of age. Here, we performed the first genome-wide scan for non-MHC IDDM susceptibility genes in this strain. The analysis of three crosses has led to the revelation of a major IDDM susceptibility gene, termed $I d d m / k d p 1$, on rat chromosome (Chr) 11. Homozygosity for the KDP allele at this locus is shown to be essential for the development of moderate to severe insulitis and the onset of IDDM. Comparative mapping suggests that the homologues of $I d d m /$ $k d p 1$ are located on human Chr 3 and mouse Chr 16 and would therefore be different from previously reported IDDM susceptibility genes. (J. Clin. Invest. 1997. 100:2015-2021.) Key words: insulin-dependent diabetes mellitus • insulitis • susceptibility genes $\bullet$ linkage analysis $\bullet$ comparative mapping
\end{abstract}

\section{Introduction}

Whole genome genetic dissection of polygenic traits has become possible recently in the human and mouse with the development of detailed genetic maps, particularly with the use of simple sequence length polymorphism (SSLP) $)^{1 / \text { microsatel- }}$ lite markers. Although the rat genetic map was rudimentary compared with those of the human and mouse, significant progress has been made in the past years (1-3).

Insulin-dependent (type I) diabetes mellitus (IDDM) is an autoimmune disease with multifactorial etiology, and it has be-

Address correspondence to Kajuro Komeda, Ph.D., Division of Laboratory Animal Science, Animal Research Center, Tokyo Medical College, Shinjuku-ku, Tokyo 160, Japan. Phone: 81-3-3351-6141; FAX: 81-3-3350-8659; E-mail: komeda@tokyo-med.ac.jp

Received for publication 4 February 1997 and accepted in revised form 27 August 1997.

J. Clin. Invest.

(C) The American Society for Clinical Investigation, Inc. 0021-9738/97/10/2015/07 \$2.00

Volume 100, Number 8, October 1997, 2015-2021

http://www.jci.org come a target of comprehensive genetic analyses in humans and animals. So far, several IDDM susceptibility genes have been mapped in these species (4-8). In the nonobese diabetic mouse, at least 15 different loci are linked to disease development $(8,9)$, and studies to identify these genes are undertaken with congenic strategy and/or the positional candidate gene approach. The BB rat is another well-studied model for IDDM. At least three genes are involved in the development of the disease: Lyp (Iddm1), which controls T cell lymphopenia, an MHC-linked gene (Iddm2), and the third gene (Iddm3) (5, 1012). Although these animal models have contributed to the understanding of IDDM, some characteristics of human IDDM are not fully covered by these models. Other animal models with characteristics different from those of the nonobese diabetic mouse and the $\mathrm{BB}$ rat are therefore essential for the elucidation of the human disorder.

The Long-Evans Tokushima Lean (LETL) rat is among the best models for this disorder. The characteristics are rapid onset of overt diabetes with no sex difference in the incidence, autoimmune destruction of pancreatic $\beta$ cells, and no significant $\mathrm{T}$ cell lymphopenia (13). The cumulative frequency of diabetes had been maintained at $<30 \%$ to avoid a transition to high frequency of diabetes, which resulted in poor reproductive performance (13). The first genetic analysis showed that at least two recessive genes are responsible for the induction of insulitis, one of which is closely linked with rat MHC RT1 ${ }^{\mathrm{u}}$ haplotype (13).

To make the LETL rat a fully usable model of IDDM, the frequency of diabetes must be elevated. Therefore, we have begun to isolate a diabetes-prone substrain from the LETL rat. Briefly, selective brother-sister mating has been conducted using diabetic animals or nondiabetic ones but with severe insulitis. The cumulative frequency of diabetes in the animals generated from diabetic parents was $70 \%$ at $120 \mathrm{~d}$ of age, and reached $82 \%$ within $220 \mathrm{~d}$ of age. It was noteworthy that all nondiabetic animals developed moderate to severe insulitis within $220 \mathrm{~d}$ of age. Since these characteristics are apparently different from those of the LETL rat, we named the diabetesprone substrain Komeda Diabetes-Prone (KDP) rat.

In this study, we report on the first genome-wide scan for non-MHC IDDM susceptibility genes in the KDP rat. The results show that a novel non-MHC gene, termed $I d d m / k d p 1$, on rat chromosome (Chr) 11 is essential for the development of moderate to severe insulitis and the onset of diabetes.

1. Abbreviations used in this paper: BN, Brown Norway rat; Chr, chromosome; IDDM, insulin-dependent diabetes mellitus; KDP, Komeda Diabetes-Prone rat; LETL, Long-Evans Tokushima Lean rat; LETO, Long-Evans Tokushima Otsuka rat; SSLP, simple sequence length polymorphism; TM, Tester Moriyama rat. 


\section{Methods}

Cross. The LETL and Long-Evans Tokushima Otsuka (LETO [13], a control strain without any diabetic symptoms derived from the same origin as the LETL) rats were obtained from Dr. K. Kawano (Tokushima Research Institute, Otsuka Pharmaceutical Co., Tokushima, Japan). The Brown Norway (BN/Sea) rat was purchased from Seiwa Experimental Animals Co. Ltd. (Fukuoka, Japan). The Tester Moriyama (TM/Kom) rat was maintained at the Animal Research Center, Tokyo Medical College. All rats were maintained under specific pathogen-free conditions with a 12-h light/dark cycle, FR-1 commercial diet (Funabashi Farm Co. Ltd., Chiba, Japan), and water ad libitum at the same facility.

The KDP rat was developed as a diabetes-prone substrain of the LETL rat by selective brother-sister mating, and only diabetic males were used to generate backcross progeny with the three reference strains, the TM, LETO, and BN rats: $(\mathrm{TM} \times \mathrm{KDP}) \mathrm{F} 1 \times \mathrm{KDP}$, $(\mathrm{LETO} \times \mathrm{KDP}) \mathrm{F} 1 \times \mathrm{KDP}$ and $(\mathrm{BN} \times \mathrm{KDP}) \mathrm{F} 1 \times \mathrm{KDP}$. All crosses were conducted under specific pathogen-free conditions in the same facility. Rat MHC RT1 haplotypes of the KDP, TM, LETO, and BN rats are $u, u, u$, and $n$, respectively. All animal care and procedures were approved by the Steering Committee of Research-Related Laboratory Animals of Tokyo Medical College.

Phenotyping. The F1 and backcross progeny were phenotyped for insulitis and diabetes as follows. Animals were checked for glycosuria three times a week by Tes-tape (Eli Lilly and Co., Indianapolis, IN) until $120 \mathrm{~d}$ of age. Nonfasting blood glucose levels of glycosuric animals ( 2 to $4+$ ) were measured on tail blood by Glutest E (Kyoto Daiichi Chemicals Co. Ltd., Kyoto, Japan). Diabetes was defined as 2 to $4+$ glycosuria and blood glucose levels $>200 \mathrm{mg} / \mathrm{dl}$ under ad libitum dietary conditions. Diabetic animals were treated with $2-5 \mathrm{U} / \mathrm{d}$ of Ultralente insulin (Novo-Nordisk Pharmaceutical Co. Ltd., Bagsvaerd, Denmark) in the evening for survival.

Four blocks from the whole pancreas of each animal were fixed in Bouin's solution. 5- $\mu \mathrm{m}$ paraffin sections were prepared and stained with hematoxylin and eosin. Light microscopic observations of the serial sections were done by examiners with no information concerning the diabetic status of the animals. First, each pancreatic islet was graded from intact to severe: an islet having no intraislet infiltration cells being considered as intact, a slightly infiltrated islet as slight insulitis, a clearly infiltrated islet as mild insulitis (area of infiltration/islet area $<25 \%$ ), a markedly infiltrated islet as moderate insulitis $(25 \%<$ area of infiltration/islet area $<75 \%)$, and a severely infiltrated islet as severe insulitis ( $75 \%<$ area of infiltration/islet area).

Then, the degree of insulitis of each animal was graded from 1 to 5 based on the percentages of moderately and severely infiltrated islets. Animals with no or slight insulitis were given a score of 1 . A score of 2 was given to those with $<10 \%$ severely and $<50 \%$ moderately infiltrated islets. A score of 3 was given to those with $<10 \%$ severely and $\geq 50 \%$ moderately infiltrated islets, with $\geq 10 \%$ but $<30 \%$ severely infiltrated islets, or with $\geq 30 \%$ but $<50 \%$ severely and $<50 \%$ moderately infiltrated islets. A score of 4 was given to those with $\geq 30 \%$ but $<50 \%$ severely and $\geq 50 \%$ moderately infiltrated islets, or with $\geq 50 \%$ but $<70 \%$ severely infiltrated islets. Those with $\geq 70 \%$ severely infiltrated islets were classified as end-stage infiltration and were given a score of 5 .

Genotyping. Genotyping was performed using SSLP markers described elsewhere $(2,3,14-19)$, with the exception of $D 20 R w h 1$ which was detected by PCR-RFLP analysis (5) and D17Kyol which was revealed by arbitrarily primed-PCR analysis (20). To genotype backcross progeny, genomic DNA ( $80 \mathrm{ng}$ ) samples were amplified by PCR. PCR conditions were as follows: $50 \mathrm{mM} \mathrm{KCl}, 20 \mathrm{mM}$ Tris- $\mathrm{HCl}(\mathrm{pH}$ 8.4), 1-2 mM MgCl $2,0.05 \% \mathrm{~W}-1$ buffer (GIBCO BRL, Gaithersburg, MD), $200 \mu \mathrm{M}$ each of dNTPs, $200 \mathrm{nM}$ each of sense and antisense primers, and $0.4 \mathrm{U}$ Taq DNA polymerase (GIBCO BRL) in a volume of $20 \mu \mathrm{l}$, overlaid with $40 \mu \mathrm{l}$ of mineral oil. Reactions were set in a flexible 96-well plate (Becton Dickinson and Co., Oxnard, CA) and amplified on a thermal cycler (MJ Research, Inc., Watertown, MA) using the following thermocycling protocol: initial denaturation at $94^{\circ} \mathrm{C}$ for $3 \mathrm{~min}$, followed by 35 cycles of $94^{\circ} \mathrm{C}$ for $30 \mathrm{~s}$, annealing for 1 min and $72^{\circ} \mathrm{C}$ for $45 \mathrm{~s}$, with a final extension period, $72^{\circ} \mathrm{C}$ for $5 \mathrm{~min}$. Optimized annealing temperatures ranged from 50 to $60^{\circ} \mathrm{C}$. PCR products were mixed with loading buffer $(0.05 \%$ bromophenol blue, $50 \%$ glycerol), electrophoresed on $4 \%$ NuSieve 3:1 agarose gels (FMC BioProducts, Rockland, ME), and stained with ethidium bromide. Although most of the alleles were detected by this type of analysis, some of them were not resolved. In this case, radiolabeled PCR products were run on sequencing gels. DNA fragments were amplified in a reaction volume of $25 \mu \mathrm{l}$ containing $100 \mathrm{ng}$ of genomic DNA, $50 \mathrm{mM} \mathrm{KCl}, 20 \mathrm{mM}$ Tris-HCl (pH 8.4), 1-2 mM MgCl $2,0.05 \% \mathrm{~W}-1$ buffer, $20 \mu \mathrm{M}$ each of dATP, dGTP, and dTTP, $2 \mu \mathrm{M}$ of dCTP, $1 \mu \mathrm{Ci}$ $\left[\alpha{ }^{32} \mathrm{P}\right] \mathrm{dCTP}(6,000 \mathrm{Ci} / \mathrm{mmol}), 200 \mathrm{nM}$ each of primers, and $0.5 \mathrm{U}$ Taq DNA polymerase, overlaid with $50 \mu \mathrm{l}$ of mineral oil. Amplifications were performed in a DNA thermal cycler (Perkin Elmer Cetus Corp., Norwalk, CT) using the thermocycling protocol as described above. After amplifications, PCR products were mixed with $20 \mu \mathrm{l}$ of formamide buffer ( $95 \%$ formamide, $0.05 \%$ bromophenol blue, $0.05 \%$ xylene cyanol, $20 \mathrm{mM}$ EDTA), heated at $94^{\circ} \mathrm{C}$ for $5 \mathrm{~min}$, cooled immediately to $4^{\circ} \mathrm{C}$, loaded on $5 \%$ polyacrylamide, $7 \mathrm{M}$ urea sequencing gels, and electrophoresed at 2,000 V for $2 \mathrm{~h}$. The gels were dried down, exposed overnight, and visualized by autoradiography.

Linkage analysis. The segregation patterns of the markers in the backcross progeny were analyzed with the Map Manager computer program (21). Linkage of markers to diabetes was evaluated by $\chi^{2}$ tests in $2 \times 2$ contingency tables.

Comparative mapping. Two mouse markers, D16Mit4 and D16Mit77, were applicable to the rat as the same primers for the mouse, whereas primers for D16Mit46 were newly designed as follows: 5'-TTTAACTTGAATTTCACTGT-3' and 5'-AGAATAATTCCAGACATCAA-3'. For genotyping by mouse SSLP markers, the same protocol was used, as described above. We genotyped $($ ALY $\times$ MSM)F1 $\times$ ALY mouse backcross sets (22) for mapping a rat marker, D11Mgh5, on mouse Chr 16. Rat Gap43 and Sod1 gene sequences were obtained from GenBank, and primers flanking microsatellite were designed as follows: Gap43, 5'-ACCTCTATGCGGTTTTAGAT-3' and 5'-AGTTACAGCTGCCAGGAGAT-3'; Sod1 1, 5'-ACAATTCCTGAATGTGTACAACTCT-3' and 5'-AGCTCTAAATAAGTCTCAGTCTCTC-3'.

\section{Results}

Inheritance of diabetes and insulitis. To investigate the genetic control of insulitis and diabetes in the KDP rat, we generated two MHC-matched backcrosses, $(\mathrm{TM} \times \mathrm{KDP}) \mathrm{F} 1 \times \mathrm{KDP}$ and $(\mathrm{LETO} \times \mathrm{KDP}) \mathrm{F} 1 \times \mathrm{KDP}($ termed TM cross and LETO cross, respectively), in which both the TM and LETO rats have the same MHC haplotype (RT1 $\left.{ }^{\mathrm{u}}\right)$ as the KDP rat. We also generated one MHC-mismatched backcross, $(\mathrm{BN} \times \mathrm{KDP}) \mathrm{F} 1 \times$ $\mathrm{KDP}$ (termed $\mathrm{BN}$ cross), in which the $\mathrm{BN}$ rat has a different MHC haplotype $\left(\mathrm{RT}^{\mathrm{n}}\right)$. In the F1 generation, most of the animals were free from insulitis, some of them showed only slight insulitis, and none of them developed overt diabetes (data not shown). The cumulative frequency of diabetes in the backcross progeny varied with the cross (Fig. 1). At $120 \mathrm{~d}$ of age, 32 and $34 \%$ of the animals in the TM and LETO crosses developed diabetes, respectively, while $16 \%$ of the animals in the $\mathrm{BN}$ cross developed diabetes. In the case of insulitis, the proportion of the animals classified as specific scores also depends on the cross. If the animals are divided into two classes, score 1 (no or slight insulitis, which corresponds to F1 type) and scores 2-5 (mild insulitis to end stage infiltration), the proportions of the animals with scores $2-5$ were $51 \%$ in the TM cross and $46 \%$ in the LETO cross, while $35 \%$ in the BN cross. 


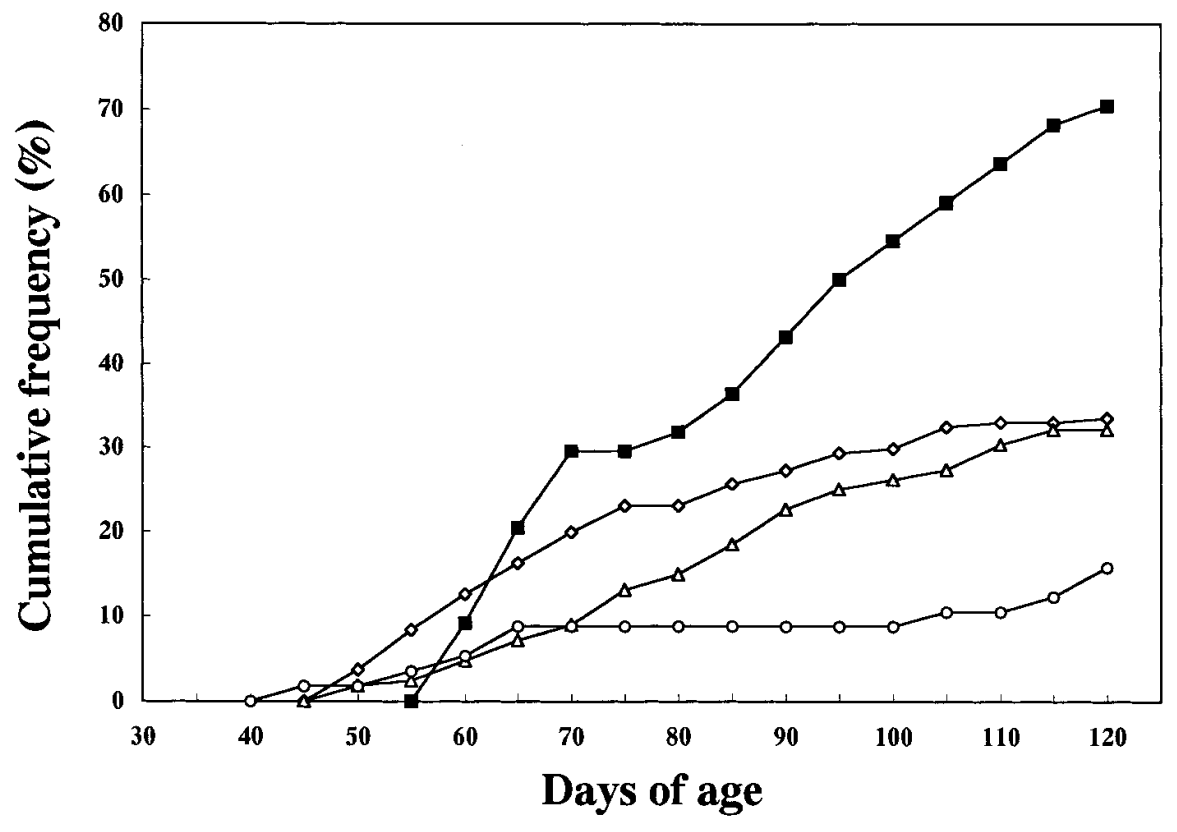

Figure 1. Cumulative frequency of diabetes in the parental KDP strain and the three backcrosses. The cumulative frequency of diabetes in the KDP strain was based on the animals generated from diabetic parents. Filled boxes, the KDP strain $(n=44)$; open diamonds, the $(\mathrm{LETO} \times \mathrm{KDP}) \mathrm{F} 1 \times \mathrm{KDP}$ backcross $(n=191)$; open triangles, the $(\mathrm{TM} \times \mathrm{KDP}) \mathrm{F} 1 \times \mathrm{KDP}$ backcross $(n=$ $168)$; open circles, the $(\mathrm{BN} \times \mathrm{KDP}) \mathrm{F} 1 \times$ KDP backcross $(n=57)$.
A genome-wide scan for IDDM susceptibility genes. In an attempt to detect non-MHC IDDM susceptibility genes, we genotyped markers throughout the rat genome on the backcross progeny from the TM cross (Table I). Since the TM cross is MHC-matched, MHC-linked genes should not segregate in this cross. In diabetic animals, two chromosomal regions, on Chr 2 (D2Mit16, $\chi^{2}=4.0, P<0.05$ ) and Chr 11 (all markers, $\left.\chi^{2}>12.4, P<0.001\right)$, showed an excess of homozygotes, while two other regions, on $\mathrm{Chr} 2\left(C p b, \chi^{2}=5.0, P<0.05\right)$ and $\mathrm{Chr}$ 18 (Gjal and Olf, $\chi^{2}=5.3$ and 4.9, respectively, $P<0.05$ ), showed an excess of heterozygotes. Except for Chr 11, these distortions of genotypes in the progeny may reflect weak evidence of linkage or random fluctuations. In contrast, we detected strong evidence of linkage to diabetes on Chr 11 markers (D11M16Mit46, $\left.\chi^{2}=55.4, P<0.001\right)$. The strong bias to homozygotes in diabetic animals suggests that there is one recessively acting diabetogenic gene in the vicinity of D11M16Mit46 locus. Assuming that this gene acts in a fully recessive manner and using the genotypes of diabetic animals only, the position of the gene, termed $I d d m / k d p 1$, was accurately mapped between Mox2 and D11M16Mit46 (Fig. 2). This means that all diabetic animals are homozygous for the KDP allele at the locus, and that $I d d m / k d p 1$ acts in a recessive manner. These results, together with the inheritance pattern of diabetes described above, suggest that the inheritance of diabetes in the TM cross could be explained by the segregation of only one gene, $I d d \mathrm{~m} /$ $k d p 1$.

To replicate these findings and to confirm the effect of an MHC-linked gene on insulitis and diabetes, we genotyped the animals generated from the other two crosses: the LETO and $\mathrm{BN}$ crosses (Table II). In these crosses, strong evidence of linkage to diabetes was found in the same region on Chr 11 (D11Mgh5 in the LETO cross, $\chi^{2}=64.8, P<0.001$; Mox2 in the BN cross, $\left.\chi^{2}=9.0, P<0.005\right)$, and no evidence of linkage was detected in the other chromosomes (data not shown), except for MHC (RT1) locus in the BN cross (D20Rwh1, $\chi^{2}=$ 5.1, $P<0.05)$. As for the TM cross, the results indicate that all diabetic animals are homozygous for the KDP allele at Iddm/
A

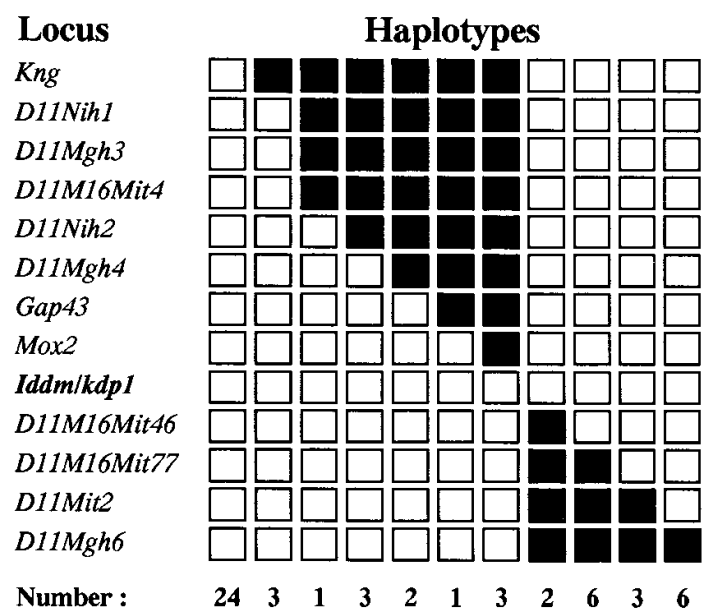

B

\section{Chromosome 11}

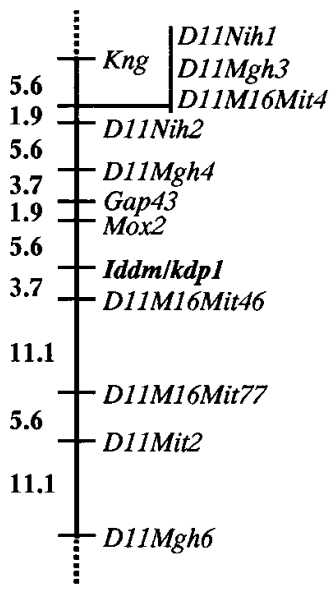

Figure 2. Mapping of $I d d m / k d p 1$. A total of 54 diabetic animals from the $(\mathrm{TM} \times$ $\mathrm{KDP}) \mathrm{F} 1 \times$ KDP backcross was used to map $I d d m / k d p 1$ by genetic linkage and haplotype analysis. $(A)$ Distribution of haplotypes for Chr 11 observed among the 54 backcross progeny. Black boxes represent heterozygosity for the TM allele. White boxes represent homozygosity for the KDP allele. $(B)$ Genetic linkage map of Chr 11 including $I d d m / k d p 1$ locus. Distances between loci are shown to the left in centiMorgans, calculated with the Map Manager program (21). D11M16Mit4, D11M16Mit46, and D11M16Mit77 are mouse $\mathrm{Chr} 16$ markers which were mapped to rat $\mathrm{Chr} 11$. 
Table I. Associations of Markers with Diabetes in the $(T M \times$ $K D P) F 1 \times K D P$ Cross

\begin{tabular}{|c|c|c|c|c|c|c|c|c|}
\hline \multirow[b]{2}{*}{$\mathrm{Chr}$} & \multirow[b]{2}{*}{ Locus name } & \multirow[b]{2}{*}{ Distance } & \multicolumn{2}{|c|}{ Diabetics } & \multicolumn{2}{|c|}{$\begin{array}{c}\text { Non- } \\
\text { diabetics }\end{array}$} & \multirow[b]{2}{*}{$x^{2}$} & \multirow[b]{2}{*}{$P$ value } \\
\hline & & & Но & $\mathrm{He}$ & Ho & $\mathrm{He}$ & & \\
\hline & & $c M$ & & & & & & \\
\hline \multirow[t]{7}{*}{1} & D1Nih2 & $46.5^{*}$ & 26 & 28 & 33 & 54 & & \\
\hline & Klk1 & $27.9^{*}$ & 20 & 23 & ND & ND & & \\
\hline & Bsis & 7.0 & 26 & 28 & 59 & 55 & & \\
\hline & Mt1pa & 7.0 & 20 & 22 & ND & ND & & \\
\hline & $\operatorname{Igf2}$ & 6.7 & 25 & 29 & 55 & 53 & & \\
\hline & Pbpc2 & 29.9 & 23 & 31 & 53 & 48 & & \\
\hline & D1Mgh12 & - & 20 & 33 & 56 & 58 & & \\
\hline \multirow[t]{8}{*}{2} & D2Mit6 & 8.4 & 18 & 36 & 50 & 52 & & \\
\hline & $C p b$ & 26.8 & 19 & 34 & 61 & 51 & 5.0 & $<0.05$ \\
\hline & D2Mit9 & 7.0 & 26 & 27 & 35 & 26 & & \\
\hline & $P k l r$ & 7.0 & 22 & 21 & ND & ND & & \\
\hline & $H s d 3 b$ & 2.4 & 27 & 27 & 66 & 48 & & \\
\hline & $C d 53$ & 7.3 & 23 & 18 & ND & ND & & \\
\hline & D2Nih5 & 23.8 & 28 & 26 & 52 & 43 & & \\
\hline & D2Mit16 & - & 35 & 19 & 54 & 58 & 4.0 & $<0.05$ \\
\hline \multirow[t]{5}{*}{3} & D3Куо2 & 11.9 & 27 & 15 & ND & ND & & \\
\hline & D3Arb178 & $39.0^{*}$ & 29 & 23 & 60 & 48 & & \\
\hline & D3Mit13 & 29.9 & 22 & 32 & 53 & 60 & & \\
\hline & Svs2 & 2.3 & 20 & 34 & 54 & 57 & & \\
\hline & Stnl & - & 15 & 29 & ND & ND & & \\
\hline \multirow[t]{4}{*}{4} & Cpal & 2.3 & 27 & 27 & 54 & 59 & & \\
\hline & Npy & $43.2 *$ & 20 & 24 & ND & ND & & \\
\hline & Eno2 & 9.1 & 20 & 34 & 52 & 60 & & \\
\hline & Pthlh & - & 17 & 27 & ND & ND & & \\
\hline \multirow[t]{5}{*}{5} & D5Mit1 & 2.3 & 21 & 23 & ND & ND & & \\
\hline & D5Mit9 & 14.5 & 26 & 27 & 59 & 53 & & \\
\hline & D5Mit11 & 25.0 & 26 & 28 & 56 & 52 & & \\
\hline & Glut1 & 2.3 & 19 & 25 & ND & ND & & \\
\hline & D5Mit13 & - & 25 & 29 & 56 & 57 & & \\
\hline \multirow[t]{5}{*}{6} & D6Cер8 & $37.2^{*}$ & 21 & 33 & 61 & 50 & & \\
\hline & D6Mit2 & 2.3 & 19 & 24 & ND & ND & & \\
\hline & D6Mit8 & 9.3 & 21 & 33 & 55 & 54 & & \\
\hline & D6Mit10 & 2.3 & 18 & 25 & ND & ND & & \\
\hline & $C k b$ & - & 17 & 27 & ND & ND & & \\
\hline \multirow[t]{8}{*}{7} & Igf1 & 37.5 & 23 & 31 & 61 & 45 & & \\
\hline & D7Cep1 & 6.8 & 20 & 34 & 60 & 54 & & \\
\hline & D7Mit5 & 9.1 & 17 & 27 & ND & $\mathrm{ND}$ & & \\
\hline & $M y c$ & 2.3 & 17 & 27 & ND & ND & & \\
\hline & D7Mit4 & 5.1 & 20 & 34 & 57 & 57 & & \\
\hline & D7Mit3 & 17.6 & 13 & 26 & ND & ND & & \\
\hline & D7Mit11 & 2.6 & 18 & 21 & ND & ND & & \\
\hline & Prph & - & 23 & 31 & 51 & 57 & & \\
\hline \multirow[t]{8}{*}{8} & D8Kyol & 4.3 & 26 & 21 & ND & ND & & \\
\hline & D8N228 & 14.3 & 30 & 24 & 53 & 61 & & \\
\hline & Ncam & 16.7 & 33 & 21 & 50 & 64 & & \\
\hline & D8N136 & 0.6 & 35 & 19 & 56 & 58 & & \\
\hline & D8Mgh4 & 16.2 & 35 & 19 & 57 & 56 & & \\
\hline & Rhp2 & 12.8 & 31 & 23 & 66 & 48 & & \\
\hline & D8Mgh1 & 3.1 & 28 & 25 & 68 & 43 & & \\
\hline & Acaa & - & 27 & 27 & ND & $\mathrm{ND}$ & & \\
\hline \multirow[t]{4}{*}{9} & $D 9 M g h 5$ & $38.2 *$ & 29 & 25 & 46 & 53 & & \\
\hline & Cryga & 2.3 & 34 & 20 & 66 & 47 & & \\
\hline & D9Mit3 & $31.8^{*}$ & 26 & 18 & ND & ND & & \\
\hline & D9Куо 2 & - & 31 & 23 & 54 & 47 & & \\
\hline
\end{tabular}

Continued
Table I. (Continued)

\begin{tabular}{|c|c|c|c|c|c|c|c|c|}
\hline \multirow[b]{2}{*}{$\mathrm{Chr}$} & \multirow[b]{2}{*}{ Locus name } & \multirow[b]{2}{*}{ Distance } & \multicolumn{2}{|c|}{ Diabetics } & \multicolumn{2}{|c|}{$\begin{array}{c}\text { Non- } \\
\text { diabetics }\end{array}$} & \multirow[b]{2}{*}{$x^{2}$} & \multirow[b]{2}{*}{$P$ value } \\
\hline & & & Ho & $\mathrm{He}$ & Но & $\mathrm{He}$ & & \\
\hline & & $c M$ & & & & & & \\
\hline \multirow[t]{5}{*}{10} & D10Mit10 & 15.9 & 23 & 31 & 44 & 44 & & \\
\hline & D10Mit9 & 13.6 & 17 & 27 & ND & ND & & \\
\hline & $S y b 2$ & 2.3 & 21 & 33 & 49 & 63 & & \\
\hline & Asgr1 & 18.2 & 18 & 26 & ND & ND & & \\
\hline & Ppy & - & 25 & 29 & 53 & 57 & & \\
\hline \multirow[t]{12}{*}{11} & Kng & 4.8 & 41 & 13 & 45 & 69 & 19.5 & $<0.001$ \\
\hline & D11Nih1 & 0.0 & 44 & 10 & 48 & 66 & 22.9 & $<0.001$ \\
\hline & $D 11 M g h 3$ & 0.0 & 44 & 10 & 48 & 66 & 22.9 & $<0.001$ \\
\hline & D11M16Mit4 & 1.2 & 44 & 10 & 36 & 61 & 27.4 & $<0.001$ \\
\hline & D11Nih2 & 4.2 & 45 & 9 & 47 & 67 & 26.2 & $<0.001$ \\
\hline & D11Mgh4 & 3.0 & 48 & 6 & 47 & 67 & 33.9 & $<0.001$ \\
\hline & Gap43 & 1.8 & 50 & 4 & 44 & 70 & 43.4 & $<0.001$ \\
\hline & Mox2 & 11.3 & 51 & 3 & 46 & 68 & 43.9 & $<0.001$ \\
\hline & D11M16Mit46 & 8.3 & 52 & 2 & 40 & 74 & 55.4 & $<0.001$ \\
\hline & D11M16Mit77 & 4.2 & 46 & 8 & 40 & 74 & 36.8 & $<0.001$ \\
\hline & D11Mit2 & 10.1 & 43 & 11 & 40 & 74 & 29.1 & $<0.001$ \\
\hline & D11Mgh6 & - & 37 & 17 & 45 & 69 & 12.4 & $<0.001$ \\
\hline \multirow[t]{5}{*}{12} & D12Mit8 & $50.0^{*}$ & 27 & 17 & ND & ND & & \\
\hline & D12Mit5 & 6.8 & 17 & 27 & ND & ND & & \\
\hline & D12Mit7 & 6.8 & 22 & 32 & 42 & 66 & & \\
\hline & D12Mit3 & 19.0 & 17 & 27 & ND & ND & & \\
\hline & D12Mit4 & - & 20 & 30 & 45 & 63 & & \\
\hline \multirow[t]{2}{*}{13} & D13Куоз & $42.0^{*}$ & 28 & 23 & 54 & 57 & & \\
\hline & D13Mgh6 & - & 22 & 24 & 39 & 57 & & \\
\hline \multirow[t]{2}{*}{14} & D14Mit2 & $42.0 *$ & 28 & 26 & 50 & 40 & & \\
\hline & D14Mgh2 & - & 24 & 30 & 53 & 60 & & \\
\hline \multirow[t]{2}{*}{15} & D15Mgh2 & $40.1 *$ & 22 & 25 & 52 & 44 & & \\
\hline & D15Mgh5 & - & 21 & 33 & 58 & 55 & & \\
\hline \multirow[t]{2}{*}{16} & D16Mit2 & 36.0 & 33 & 20 & 56 & 54 & & \\
\hline & D16Cер2 & - & 25 & 29 & 45 & 56 & & \\
\hline \multirow[t]{4}{*}{17} & Chrm3 & 2.2 & 29 & 25 & 52 & 60 & & \\
\hline & D17Mit3 & 16.3 & 27 & 19 & ND & ND & & \\
\hline & D17Kyo1 & 27.3 & 27 & 17 & ND & ND & & \\
\hline & $R p 135 p$ & - & 27 & 27 & 57 & 53 & & \\
\hline \multirow[t]{10}{*}{18} & D18Kyo1 & 4.5 & 18 & 26 & ND & ND & & \\
\hline & D18Mit1 & 1.2 & 19 & 35 & 52 & 62 & & \\
\hline & D18Mit2 & 8.4 & 20 & 34 & 51 & 63 & & \\
\hline & D18Mit4 & 2.4 & 18 & 36 & 50 & 63 & & \\
\hline & Tilp & 4.5 & 17 & 37 & 49 & 65 & & \\
\hline & D18Mgh1 & 14.3 & 19 & 35 & 49 & 65 & & \\
\hline & Gjal & 0.6 & 15 & 39 & 53 & 61 & 5.3 & $<0.05$ \\
\hline & Olf & 6.3 & 15 & 39 & 52 & 62 & 4.9 & $<0.05$ \\
\hline & D18Mgh3 & 9.0 & 18 & 36 & 47 & 66 & & \\
\hline & D18Mit9 & - & 17 & 37 & 49 & 65 & & \\
\hline \multirow[t]{3}{*}{19} & Hmox1 & 28.7 & 29 & 25 & 63 & 48 & & \\
\hline & D19Mit7 & 15.7 & 23 & 30 & 63 & 51 & & \\
\hline & D19Куоз & - & 21 & 31 & 55 & 53 & & \\
\hline \multirow[t]{2}{*}{20} & D20Mgh5 & 29.4 & 32 & 21 & 54 & 56 & & \\
\hline & D20Arb234 & - & 32 & 22 & 54 & 57 & & \\
\hline $\mathrm{X}$ & DXMit1 & 25.4 & 27 & 19 & 47 & 54 & & \\
\hline & DXMit4 & - & 26 & 21 & 43 & 53 & & \\
\hline
\end{tabular}

$\mathrm{Ho}$, homozygote; $\mathrm{He}$, heterozygote; $\mathrm{ND}$, not done. Linkage of markers to diabetes was evaluated by $\chi^{2}$ tests in $2 \times 2$ contingency tables. Only $\chi^{2} \geq 4$ values are shown. Distances between loci are described in centiMorgans, calculated with the Map Manager program (21). *The distance is not supported by a logarithm of odds of 2.0 or higher. 
Table II. Associations of Markers with Diabetes in the $(L E T O \times K D P) F 1 \times K D P$ and $(B N \times K D P) F 1 \times$ KDP Crosses

\begin{tabular}{|c|c|c|c|c|c|c|c|c|}
\hline \multirow[b]{2}{*}{$\mathrm{Chr}$} & \multirow[b]{2}{*}{ Locus name } & \multirow[b]{2}{*}{ Distance } & \multicolumn{2}{|c|}{ Diabetics } & \multicolumn{2}{|c|}{$\begin{array}{c}\text { Non- } \\
\text { diabetics }\end{array}$} & \multirow[b]{2}{*}{$x^{2}$} & \multirow[b]{2}{*}{$P$ value } \\
\hline & & & Ho & $\mathrm{He}$ & Ho & $\mathrm{He}$ & & \\
\hline & & $c M$ & & & & & & \\
\hline \multicolumn{9}{|c|}{$(\mathrm{LETO} \times \mathrm{KDP}) \mathrm{F} 1 \times \mathrm{KDP}$} \\
\hline \multirow[t]{2}{*}{2} & Prlr & $48.8^{*}$ & 34 & 26 & 59 & 59 & & \\
\hline & D2Mit16 & - & 30 & 28 & 58 & 60 & & \\
\hline \multirow[t]{4}{*}{11} & Sst & 27.0 & 53 & 11 & 58 & 67 & 23.2 & $<0.001$ \\
\hline & D11Mgh5 & 6.3 & 61 & 3 & 43 & 84 & 64.8 & $<0.001$ \\
\hline & D11Mit2 & 5.8 & 57 & 7 & 47 & 80 & 46.5 & $<0.001$ \\
\hline & D11Mit4 & - & 52 & 12 & 43 & 83 & 37.7 & $<0.001$ \\
\hline 18 & Tilp & - & 36 & 28 & 63 & 63 & & \\
\hline 20 & Tnfa & - & 26 & 38 & 74 & 53 & & \\
\hline \multicolumn{9}{|c|}{$(\mathrm{BN} \times \mathrm{KDP}) \mathrm{F} 1 \times \mathrm{KDP}$} \\
\hline \multirow[t]{2}{*}{2} & $C p b$ & $49.1 *$ & 3 & 6 & 26 & 20 & & \\
\hline & D2N91 & - & 6 & 3 & 24 & 23 & & \\
\hline \multirow[t]{8}{*}{11} & Sst & 0.0 & 8 & 1 & 24 & 24 & 4.7 & $<0.05$ \\
\hline & Kng & 5.3 & 8 & 1 & 24 & 24 & 4.7 & $<0.05$ \\
\hline & D11Nih1 & 7.0 & 9 & 0 & 22 & 26 & 9.0 & $<0.005$ \\
\hline & Gap43 & 0.0 & 9 & 0 & 22 & 26 & 9.0 & $<0.005$ \\
\hline & Mox 2 & 19.3 & 9 & 0 & 22 & 26 & 9.0 & $<0.005$ \\
\hline & D11Mit2 & 0.0 & 7 & 2 & 15 & 33 & 6.9 & $<0.01$ \\
\hline & Sod1 & 10.5 & 7 & 2 & 15 & 33 & 6.9 & $<0.01$ \\
\hline & D11Mit4 & - & 6 & 3 & 16 & 32 & & \\
\hline 18 & Gjal & - & 6 & 3 & 25 & 23 & & \\
\hline \multirow[t]{5}{*}{20} & D20Rwh1(RT1) & 1.8 & 8 & 1 & 23 & 25 & 5.1 & $<0.05$ \\
\hline & D20Mgh5 & 11.5 & 8 & 1 & 24 & 24 & 4.7 & $<0.05$ \\
\hline & D20Arb548 & 17.3 & 7 & 2 & 20 & 23 & & \\
\hline & Prkacn2 & 14.0 & 5 & 4 & 24 & 24 & & \\
\hline & D20Mgh1 & - & 4 & 5 & 25 & 23 & & \\
\hline
\end{tabular}

$\mathrm{Ho}$, homozygote; $\mathrm{He}$, heterozygote. Linkage of markers to diabetes was evaluated by $\chi^{2}$ tests in $2 \times 2$ contingency tables. Only $\chi^{2} \geq 4$ values are shown. Distances between loci are described in centiMorgans, calculated with the Map Manager program (21). *The distance is not supported by a logarithm of odds of 2.0 or higher.

$k d p 1$ locus (Fig. 3), and that $I d d m / k d p 1$ acts in a recessive manner. In the $\mathrm{BN}$ cross, eight diabetic animals out of nine were homozygous for the KDP allele at MHC (Table II and Fig. 3), suggesting that an MHC-linked gene would be associated with diabetes and acts in a mostly recessive manner. These results demonstrate that $I d d m / k d p 1$ is a major diabetogenic factor in the KDP rat irrespective of the cross, and that the inheritance of diabetes could be explained by the segregation of $I d d m / k d p 1$ in the MHC-matched crosses, the TM and LETO crosses, and by the segregation of $I d d m / k d p 1$ and an MHC-linked gene in the MHC-mismatched cross, the $\mathrm{BN}$ cross.

The influence of Iddm/kdp1 on the development of insulitis. To reveal the effect of $I d d m / k d p 1$ in the pathogenesis of diabetes, we investigated the influence of $I d d m / k d p 1$ on insulitis (Table III). Since all diabetic animals showed end-stage infiltration (score 5), association between insulitis scores in nondiabetic animals and genotypes at the closest markers to $I d d m /$ $k d p 1$ locus was examined. In all three crosses, a significant
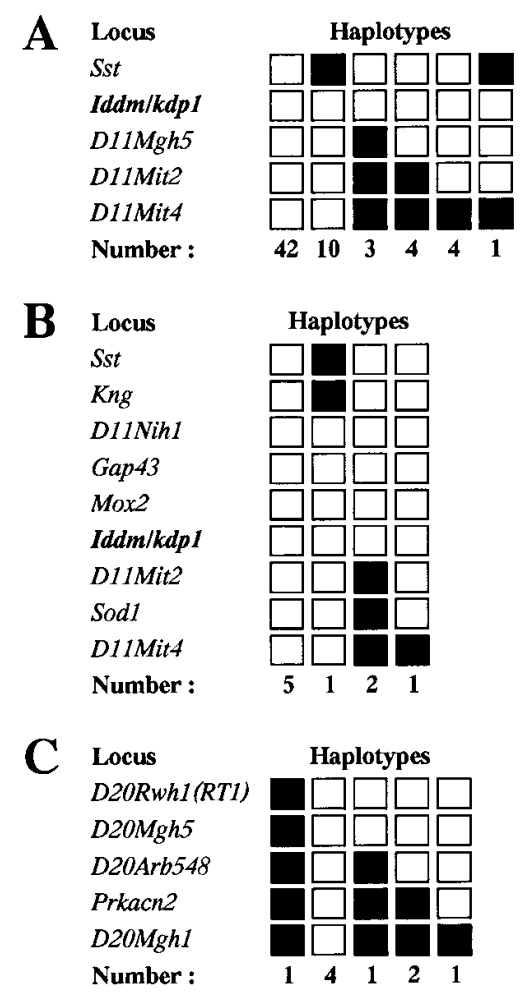

Figure 3. Distribution of haplotypes for Chr 11 including $I d d m / k d p 1$ and for Chr 20 including MHC (RT1). $(A)$ Distribution of haplotypes for Chr 11 observed among the 64 diabetic animals from the $($ LETO $\times$ KDP $)$ F1 $\times$

KDP backcross. The order of loci was confirmed by the Map Manager program (21). Black boxes represent heterozygosity for the LETO allele. White boxes represent homozygosity for the KDP allele. (B) Distribution of haplotypes for Chr 11 observed among the nine diabetic animals from the $(\mathrm{BN} \times$ KDP)F1 $\times$ KDP backcross. Except for Iddm/ $k d p 1$, the order of loci was calculated with the Map Manager program (21) by using all back-

cross progeny $(n=57)$ from the cross. The position of $I d d m / k d p 1$ was deduced from the result obtained in the TM cross (Fig. 2). Black boxes represent heterozygosity for the $\mathrm{BN}$ allele. White boxes represent homozygosity for the KDP allele. (C) Distribution of haplotypes for $\mathrm{Chr} 20$ observed among the nine diabetic animals from the $(\mathrm{BN} \times$ KDP)F1 $\times$ KDP backcross. The order of loci was calculated with the Map Manager program (21) by using all backcross progeny $(n=57)$ from the cross. Black boxes represent heterozygosity for the BN allele. White boxes represent homozygosity for the KDP allele.

number of nondiabetic animals homozygous for the KDP allele developed moderate to severe insulitis (scores 3-4), whereas those heterozygous remained intact or had mild insulitis (scores 1-2), with the only exception of one animal in the TM cross which developed moderate insulitis. These findings indicate that homozygosity for the KDP allele at $I d d m / k d p 1$ locus is strongly associated with the development of moderate to severe insulitis, and that $I d d m / k d p 1$ acts in a recessive manner. Thus, it is suggested that $I d d m / k d p 1$ would influence the development of insulitis in the pathogenesis of diabetes.

Comparative mapping of Iddm/kdp1 region among the rat, mouse, and human. Next, we conducted comparative mapping of $I d d m / k d p 1$ region among the rat, mouse, and human, to make clear as to whether the homologues of $I d d m / k d p 1$ have been mapped in the human and mouse. This has been done by reciprocal use of SSLP markers between the mouse and rat (23), and by development of rat gene markers. Based on the synteny information (24), we screened $\sim 100$ SSLP markers on mouse $\mathrm{Chr} 16$, and detected three markers, D16Mit4, D16Mit46, and D16Mit77, which could be applicable in the TM cross. As shown in Fig. 4, those markers were successfully mapped on rat Chr 11 . Conversely, we mapped a rat SSLP marker, D11Mgh5, on mouse Chr 16. Large segments of mouse Chr 16 have been shown to be homologous to human 
Table III. Association of Iddm/kdp1 Genotypes with the Frequency and Severity of Insulitis

\begin{tabular}{|c|c|c|c|c|c|c|}
\hline \multirow[b]{2}{*}{ Cross } & \multirow[b]{2}{*}{$\begin{array}{l}I d d m / k d p 1 \\
\text { genotype }\end{array}$} & \multicolumn{4}{|c|}{ Insulitis score } & \multirow[b]{2}{*}{$\begin{array}{l}\text { Mann-Whitney } \\
\text { U test }\end{array}$} \\
\hline & & 1 & 2 & 3 & 4 & \\
\hline \multirow[t]{2}{*}{$(\mathrm{TM} \times \mathrm{KDP}) \mathrm{F} 1 \times \mathrm{KDP}$} & Ho $(n=40)$ & $13(33 \%)$ & $5(13 \%)$ & $13(33 \%)$ & $9(23 \%)$ & \multirow[t]{2}{*}{$P<0.005$} \\
\hline & $\mathrm{He}(n=74)$ & $69(93 \%)$ & $4(5 \%)$ & $1(1 \%)$ & $0(0 \%)$ & \\
\hline \multirow[t]{2}{*}{$(\mathrm{LETO} \times \mathrm{KDP}) \mathrm{F} 1 \times \mathrm{KDP}$} & Ho $(n=43)$ & $23(53 \%)$ & $7(16 \%)$ & $7(16 \%)$ & $6(14 \%)$ & \multirow[t]{2}{*}{$P<0.005$} \\
\hline & $\mathrm{He}(n=84)$ & $80(95 \%)$ & $4(5 \%)$ & $0(0 \%)$ & $0(0 \%)$ & \\
\hline \multirow[t]{2}{*}{$(\mathrm{BN} \times \mathrm{KDP}) \mathrm{F} 1 \times \mathrm{KDP}$} & Ho $(n=22)$ & $13(59 \%)$ & $1(5 \%)$ & $7(32 \%)$ & $1(5 \%)$ & \multirow[t]{2}{*}{$P<0.05$} \\
\hline & $\mathrm{He}(n=26)$ & $24(92 \%)$ & $2(8 \%)$ & $0(0 \%)$ & $0(0 \%)$ & \\
\hline
\end{tabular}

Differences in the frequencies of animals with $I d d m / k d p 1$ genotypes of KDP homozygous $(\mathrm{Ho})$ and heterozygous $(\mathrm{He})$ in the insulitis scores were evaluated by Mann-Whitney U tests. Genotypes for $I d d m / k d p 1$ locus are described as the genotypes at the closest markers: D11M16Mit46 for the TM cross, D11Mgh5 for the LETO cross, and Mox 2 for the BN cross. Animals were aged to $120 \mathrm{~d}$ and insulitis scores for each animal were graded from 1 to 5 based on the percentages of moderately and severely infiltrated islets (see Methods for details). A score of 1 was given to animals with no or slight insulitis. Those with mild, moderate, or severe insulitis were given scores of 2, 3, or 4, respectively. Diabetic animals were classified as end-stage infiltration and given a score of 5 . Since all diabetic animals were given a score of 5 , only nondiabetic animals are listed here.

Chr 3 or Chr 21 (24). To clarify the synteny between rat Chr 11 and the human homologous regions, we mapped rat homologues of human Chr 3 or Chr 21 genes. These results, together with reported map locations of the human and mouse loci (24), suggest that the homologues of $I d d m / k d p 1$ are located in the region between Gap43 and D16Mit46 on mouse Chr 16 and in the human Chr 3p11-q13 region (Fig. 4).

\section{Discussion}

In this study, we performed the first genome-wide scan for non-MHC IDDM susceptibility genes in the KDP rat. Based on the genetic analysis of the three crosses, we mapped a nonMHC insulitis and IDDM susceptibility gene, $I d d m / k d p 1$, to rat $\mathrm{Chr} 11$, and showed that $I d d m / k d p 1$ acts in a recessive fashion. None of the heterozygous animals for the KDP allele at this locus appeared to develop moderate to severe insulitis, implying that homozygosity for the KDP allele at this locus would be essential for the development of moderate to severe insulitis and also for the onset of diabetes. However, a significant number of KDP homozygotes remained intact or had mild insulitis. This could be mainly due to the relatively short testing period used in this study. Therefore, at present, it was not clear if KDP homozygosity at $I d d m / k d p 1$ locus is sufficient for the development of moderate to severe insulitis and the onset of diabetes. To clarify this issue, further study is needed, in which the testing period must be lengthened. Among the reported IDDM susceptibility genes in the rat, there are two non-MHC genes, Lyp (Iddml) on Chr 4 (5) and Iddm3 on Chr 18 (11), involved in the pathogenesis of IDDM in the BB rat. The map position of $I d d m / k d p 1$ clearly demonstrates that $I d d m / k d p 1$ is different from the two.

Comparative mapping among the rat and "map-rich" species such as the human and mouse would give us mapping information of the homologues of relevant genes or mutations. Even if the homologues have not been mapped in the human and mouse, it might be possible to detect potential candidate genes. Therefore, we conducted comparative mapping of Iddm/ $k d p 1$ region among the rat, mouse, and human. The result shows that the homologues of $I d d m / k d p 1$ would be located in the human Chr 3p11-q13 region and in the region between Gap43 and D16Mit46 on mouse Chr 16, in which none of the IDDM susceptibility genes have been mapped. This indicates that $I d d m / k d p 1$ is a novel IDDM susceptibility gene. In the Iddm/

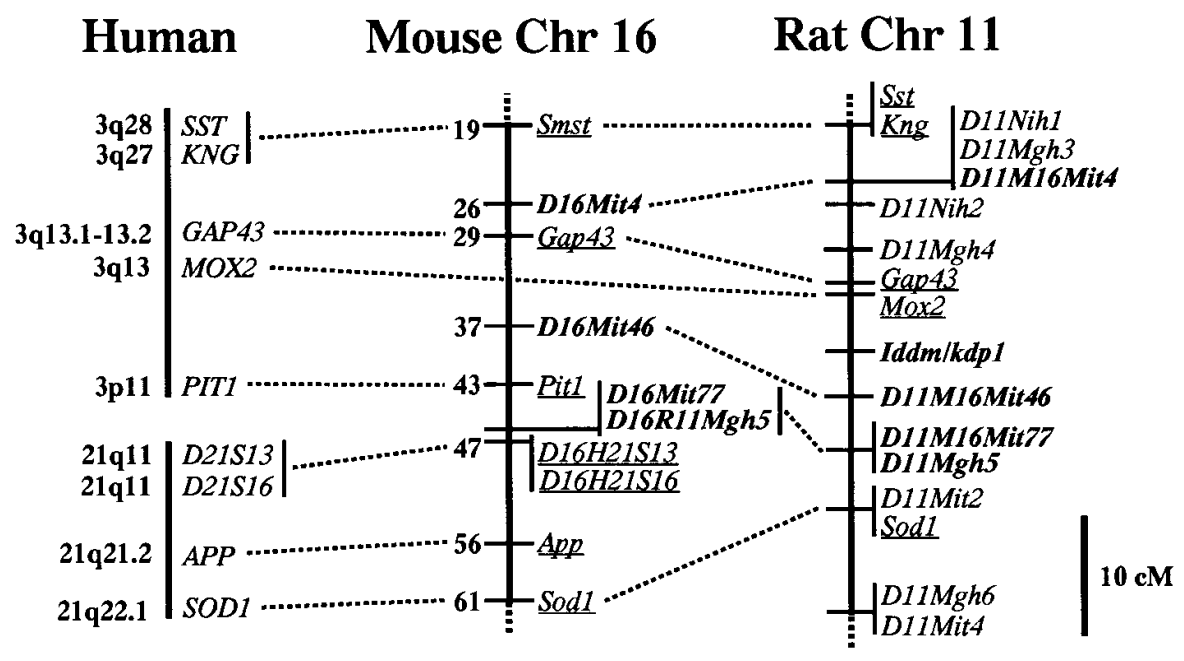

Figure 4. Comparative mapping of $I d d m /$ $k d p 1$ region among the rat, mouse, and human. The rat map is a composite map constructed from data obtained in this study, calculated with the Map Manager program (21). For the underlined loci, the map locations of the human homologues are listed to the left of the mouse map. The reciprocally used markers between the rat and mouse are indicated with bold characters. D16R11Mgh5 is a rat Chr 11 marker which was mapped to mouse Chr 16. Distances (in centiMorgans) from centromere for the mouse loci and the map locations of the human loci are based on the Mouse Genome Database (24). The bar shown at the bottom right describes genetic distances of $10 \mathrm{cM}$, which fits for both the mouse and rat maps. 
$k d p 1$ homologous region in the human and mouse, there are some potential candidate genes, relevant mutations, or, to say the least, immunologically relevant genes. Aod1, involved in neonatal thymectomy-induced autoimmune ovarian dysgenesis, has been mapped on mouse Chr 16 (25). The map position of Aodl seemed to be proximal to Gap43 (25), while Iddm/ $k d p 1$ would be located in the region distal to Gap43, which weakened a possible relationship between Aod1 and Iddm/ $k d p 1$. The others include Ly7 (lymphocyte antigen-7), CD80 (CD80 antigen, also known as CD28 antigen ligand 1 or B7-1 antigen), CD47 (CD47 antigen, also known as Rh-related antigen, integrin-associated protein, or MER6 antigen identified by $\mathrm{mAb} 1 \mathrm{D} 8$ ), and MOX2 (antigen identified by mAb MRC OX-2) $(24,26)$. The present linkage and comparative maps will help us to apply a positional candidate gene approach to clarify whether these genes are involved in the pathogenesis of IDDM in the KDP rat.

We generated the $\mathrm{BN}$ cross not only to replicate findings obtained from the TM cross, but to reveal the mode of inheritance of both $I d d m / k d p 1$ and an MHC-linked gene. In the BN cross, the animals homozygous for the KDP allele at the closest marker flanking $I d d m / k d p 1$ locus with the MHC genotypes $\mathrm{u} / \mathrm{u}$ and $\mathrm{u} / \mathrm{n}$ developed moderate to severe insulitis in 14 out of 16 cases and 3 out of 15 cases, respectively $(P<0.001$ with Fisher's exact test), and developed overt diabetes in 8 out of 16 cases and 1 out of 15 cases, respectively $(P=0.01$ with Fisher's exact test). In contrast, none of the animals heterozygous for the KDP allele at $I d d m / k d p 1$ locus developed moderate to severe insulitis. These findings indicate that the two genes, Iddm/ $k d p 1$ and an MHC-linked gene, would contribute to most of the predisposition to insulitis and diabetes in the BN cross.

Although the present linkage and comparative maps including $I d d m / k d p 1$ are relatively sparse, further detailed mapping could be performed with the help of newly developed markers. In the near future, a positional cloning or positional candidate gene approach should be applied to $I d d m / k d p 1$. Furthermore, congenic strains with the KDP allele on different genetic backgrounds should be generated to facilitate detailed genetic studies. Since $I d d m / k d p 1$ is one of the strongest diabetogenic factors ever reported, the identification and elucidation of $I d d m / k d p 1$ would contribute to the understanding, prevention, and treatment of autoimmune diseases, including IDDM.

\section{Acknowledgments}

We thank K. Shimosato, E. Oriyama, M. Namae, H. Iwama, E. Ikegaki, and R. Yagi for their technical assistance, and Dr. X. Montagutelli and Dr. T. Awata for helpful comments on the manuscript.

This study was supported in part by grants from the Ministry of Education, Science and Culture, the Ministry of Health and Welfare in Japan, Japan Foundation for Diabetes, the Epilepsy Research Foundation in Japan, the Japan Health Science Foundation, and the Japan Foundation for Aging and Health.

\section{References}

1. Levan, G., J. Szpirer, C. Szpirer, K. Klinga, C. Hanson, and M.Q. Islam. 1991. The gene map of the Norway rat (Rattus norvegicus) and comparative mapping with mouse and man. Genomics. 10:699-718.

2. Serikawa, T., T. Kuramoto, P. Hilbert, M. Mori, J. Yamada, C.J. Dubay, K. Lindpainter, D. Ganten, J.-L. Guénet, G.M. Lathrop, and J.S. Beckmann. 1992. Rat gene mapping using PCR-analyzed microsatellites. Genetics. 131: 701-721.
3. Jacob, H.J., D.M. Brown, R.K. Bunker, M.J. Daly, V.J. Dzau, A. Goodman, G. Koike, V. Kren, T. Kurtz, A. Lernmark, et al. 1995. A genetic linkage map of the laboratory rat, Rattus norvegicus. Nat. Genet. 9:63-69.

4. Todd, J.A., T.J. Aitman, R.J. Cornall, S. Ghosh, J.R.S. Hall, C.M. Hearne, A.M. Knight, J.M. Love, M.A. McAleer, J.-B. Prins, et al. 1991. Genetic analysis of autoimmune type 1 diabetes mellitus in mice. Nature (Lond.). 351:542-547.

5. Jacob, H.J., A. Pettersson, D. Wilson, Y. Mao, Å. Lernmark, and E.S Lander. 1992. Genetic dissection of autoimmune type I diabetes in the BB rat. Nat. Genet. 2:56-60.

6. Ghosh, S., S.M. Palmer, N.R. Rodrigues, H.J. Cordell, C.M. Hearne, R.J. Cornall, J.-B. Prins, P. McShane, G.M. Lathrop, L.B. Peterson, et al. 1993. Polygenic control of autoimmune diabetes in nonobese diabetic mice. Nat. Genet. 4: 404-409.

7. Davies, J.L., Y. Kawaguchi, S.T. Bennett, J.B. Copeman, H.J. Cordell, L.E. Pritchard, P.W. Reed, S.C.L. Gough, S.C. Jenkins, S.M. Palmer, et al. 1994. A genome-wide search for human type 1 diabetes susceptibility genes. Nature (Lond.). 371:130-136.

8. Wicker, L.S., J.A. Todd, and L.B. Peterson. 1995. Genetic control of autoimmune diabetes in the NOD mouse. Annu. Rev. Immunol. 13:179-200.

9. McAleer, M.A., P. Reifsnyder, S.M. Palmer, M. Prochazka, J.M. Love, J.B. Copeman, E.E. Powell, N.R. Rodrigues, J.-B. Prins, D.V. Serreze, et al. 1995. Crosses of NOD mice with the related NON strain: a polygenic model for IDDM. Diabetes. 44:1186-1195.

10. Colle, E., R.D. Guttmann, T.A. Seemayer, and F. Michel. 1983. Spontaneous diabetes mellitus syndrome in the rat. IV. Immunogenetic interactions of MHC and non-MHC components of the syndrome. Metabolism. 32:54-61.

11. Klöting, I., L. Vogt, and T. Serikawa. 1995. Locus on chromosome 18 cosegregates with diabetes in the BB/OK rat subline. Diabetes Metab. 21:338-344.

12. Awata, T., D.L. Guberski, and A.A. Like. 1995. Genetics of the BB rat: association of autoimmune disorders (diabetes, insulitis, and thyroiditis) with lymphopenia and major histocompatibility complex class II. Endocrinology. 136:5731-5735.

13. Kawano, K., T. Hirashima, S. Mori, Y. Saitoh, M. Kurosumi, and T. Natori. 1991. New inbred strain of Long-Evans Tokushima Lean rats with IDDM without lymphopenia. Diabetes. 40:1375-1381.

14. Goldmuntz, E.A., E.F. Remmers, H. Zha, J.M. Cash, P. Mathern, L.J. Crofford, and R.L. Wilder. 1993. Genetic map of 12 polymorphic loci on rat chromosome 1. Genomics. 16:761-764.

15. Zha, H., R.L. Wilder, E.A. Goldmuntz, J.M. Cash, L.J. Crofford, P. Mathern, and E.F. Remmers. 1993. Linkage map of 10 polymorphic markers on rat chromosome 2. Cytogenet. Cell Genet. 63:117-122.

16. Mathern, P., E.A. Goldmuntz, Y. Du, H. Zha, J.M. Cash, L.J. Crofford, R.L. Wilder, and E.F. Remmers. 1994. Nine polymorphic markers characterized by polymerase chain reaction techniques form two linkage groups on rat chromosome 8. Cytogenet. Cell Genet. 66:283-286.

17. Du, Y., E.F. Remmers, E.A. Goldmuntz, H. Zha, P. Mathern, L.J. Crofford, and R.L. Wilder. 1994. Simple sequence repeat length polymorphisms mapped to rat chromosome 11. Cytogenet. Cell Genet. 65:186-189.

18. Remmers, E.F., Y. Du, H. Zha, E.A. Goldmuntz, and R.L. Wilder. 1995. Ten polymorphic DNA loci, including five in the rat MHC (RT1) region, form a single linkage group on rat chromosome 20. Immunogenetics. 41:316-319.

19. Yokoi, N., K. Kitada, T. Kuramoto, T. Maihara, Y. Andoh, S. Takami, J. Higaki, T. Miki, T. Ogihara, G.M. Lathrop, and T. Serikawa. 1996. Mapping of 20 polymorphic DNA markers in the rat by somatic hybrid and linkage analysis. Mamm. Genome. 7:71-73.

20. Kuramoto, T., T. Mashimo, and T. Serikawa. 1995. Fourteen anonymous DNA markers of laboratory rats identified with arbitrarily primed polymerase chain reaction (AP-PCR). Exp. Anim. (Tokyo). 44:119-125.

21. Manly, K.F. 1993. A Macintosh program for storage and analysis of experimental genetic mapping data. Mamm. Genome. 4:303-313.

22. Kuramoto, T., T. Mashimo, R. Koike, S. Miyawaki, J. Yamada, M. Miyasaka, and T. Serikawa. 1994. The alymphoplasia (aly) mutation co-segregates with the intercellular adhesion molecule-2 (Icam-2) on mouse chromosome 11. Int. Immunol. 6:991-994.

23. Kondo, Y., M. Mori, T. Kuramoto, J. Yamada, J.S. Beckmann, D. Simon-Chazottes, X. Montagutelli, J.-L. Guénet, and T. Serikawa. 1993. DNA segments mapped by reciprocal use of microsatellite primers between mouse and rat. Mamm. Genome. 4:571-576.

24. Mouse Genome Database (MGD). Mouse Genome Informatics Project, The Jackson Laboratory, Bar Harbor, ME. World Wide Web (URL: http:// www.informatics.jax.org/mgd.html.)

25. Wardell, B.B., S.D. Michael, K.S.K. Tung, J.A. Todd, E.P. Blankenhorn, K. McEntee, J.D. Sudweeks, W.K. Hansen, N.D. Meeker, J.S. Griffith, et al. 1995. Aod1, the immunoregulatory locus controlling abrogation of tolerance in neonatal thymectomy-induced autoimmune ovarian dysgenesis, maps to mouse chromosome 16. Proc. Natl. Acad. Sci. USA. 92:4758-4762.

26. Genome Data Base (GDB). An international collaboration in support of the Human Genome Project, Johns Hopkins University School of Medicine, Baltimore, MD. World Wide Web (URL: http://gdbwww.gdb.org). 